\title{
Measurement of beauty production from dimuon events at HERA
}

\author{
P. J. Bussey \\ School of Physics and Astronomy \\ University of Glasgow \\ Glasgow, United Kingdom, G12 8QQ \\ E-mail: peter.busseyeglasgow.ac.uk
}

for the ZEUS Collaboration

Beauty production in events containing two final-state muons has been measuredwith the ZEUS detector at HERA using an integrated luminosity of $377 \mathrm{pb}^{-1}$. A low transverse-momentum threshold for muon identification, together with the large rapidity coverage of the ZEUS muon system and the upgraded ZEUS tracker, give access to an almost full phase space for beauty production in this channel. The cross section for beauty production in ep collisions has been measured to be $11.4 \pm 0.8$ (stat.) ${ }_{-3.4}^{+3.9}$ (syst.) nb. Differential cross sections and $b \bar{b}$ correlations are compared to previous measurements and theoretical models.

The 39th International Conference on High Energy Physics (ICHEP2018)

4-11 July, 2018

Seoul, Korea 
Because of their large mass, the production of $b$ quark pairs in high energy hadronic processes is governed by perturbative QCD for all transverse momenta of the $b$ quarks. This makes such processes a good area in which to test theoretical models. The HERA collider recorded $e^{ \pm} p$ interactions during two periods: 1992-2000 (HERA-I) and 2002-2007 (HERA-II). The study presented here is of beauty production using the ZEUS detector at HERA-II, in which $27.5 \mathrm{GeV} e^{ \pm}$leptons collided with $920 \mathrm{GeV}$ protons. The analysis method was similar to that of an earlier HERA-I analysis [1], but a factor of three in integrated luminosity was obtained. Events were selected in which two outgoing muons were observed, from which the cross section for the inclusive process $e^{ \pm} b \bar{b} X$ was evaluated. The entire range of exchanged photon virtualities was accepted, with no discrimination between photoproduction and deep inelastic scattering.

The use of muons as the basic signature for beauty production has as its major advantage the fact that ZEUS had close to complete angular coverage in its muon detection system, and muons of fairly low momentum could be identified. The muons are in practice associated with jets, but no jet requirement was made in the event selection and analysis. A $b \bar{b}$ event can contain several muons, deriving either directly from $b$ decay ( $\bar{b}$ will also be implied here) or indirectly through a direct decay into charm or light-flavour particles. Events with precisely two identified muons were used, and the mass of the muon pair was evaluated. For lower dimuon masses $(<4 \mathrm{GeV})$ muon pairs come from the same original $b$ and have unlike sign, while for higher masses, both likeand unlike-sign muons come from different $b$ or secondary $c$ sources. This can be seen from the illustration given in figure 1. For higher masses, unlike-sign muons also come from direct charmpair production. Light-flavour backgrounds can exist with both like- and unlike-sign muon pairs at all dimuon masses; it should be noted that at low dimuon masses, like-sign muons come only from light-flavour backgrounds.

Events were selected in which there was a total measured transverse energy of at least $8 \mathrm{GeV}$ (excluding the scattered beam lepton), standard ZEUS muon identification, and a requirement that the muon pairs should not be isolated, so as to remove Bethe-Heitler and primary $J / \psi$ events. For

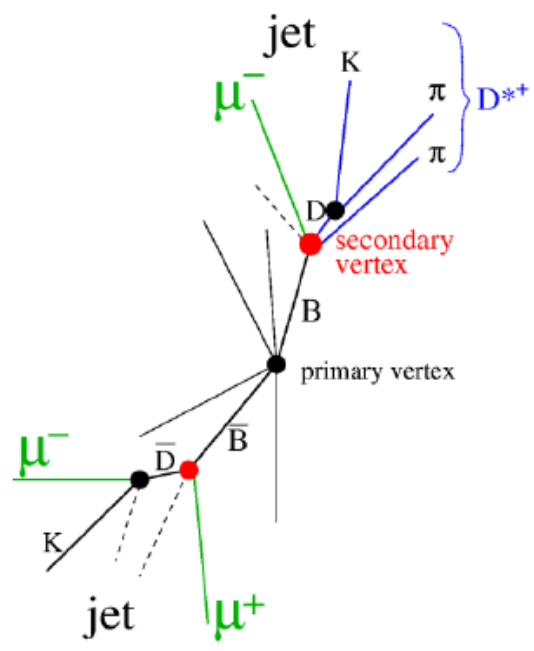

Figure 1: Schematic diagram of the decay of a $b \bar{b}$ system into several final-state muons. 
high-momentum muons a detection above $80 \%$ was achieved for the muons. Depending on the location of the muon detectors in ZEUS, the muons needed to have a transverse momentum of typically above $1.5 \mathrm{GeV}$.

To evaluate acceptances and backgrounds, photoproduction and DIS processes were simulated using the PYTHIA and RAPGAP Monte Carlo programs, respectively, and inelastic quarkonium states were simulated using HERWIG. Bethe-Heitler and exclusive quarkonium backgrounds were simulated using GRAPE-COMPTON. NLO QCD predictions were obtained using FMNR [2] linked to PYTHIA, while HVQDIS [3] at parton level was used for DIS processes, which contribute at the $15 \%$ level. The quark mass and factorisation/renormalisation scales were varied so as to give the uncertainties on the theory. Background processes were evaluated from the Monte-Carlo simulations and subtracted from the observed data, apart from the light-flavour backgrounds. The latter were evaluated experimentally from the like-sign dimuon events, with other contributions subtracted out, and were then taken to be the same for the unlike-sign dimuon events.

After the above corrections, values of the difference between the numbers of unlike-sign and like-sign events were evaluated in order to remove backgrounds from falsely identified muons, which were assumed to be the same for each case. These difference values were then converted to a set of full cross sections for beauty production. The cross sections were evaluated for beauty events giving rise to two muons in the pseudorapidity range -2.2 to +2.5 and momenta corresponding to the acceptance of the muon detectors. Results for differential cross sections are shown in figure 2. PYTHIA/RAPGAP describe the shapes of the data well, confirming their validity for evaluating the acceptance calculations, and there is also agreement with the earlier HERA-I results. An appropriate combination of FMNR and HVQDIS describes the shapes of the distributions well but seems to be somewhat low overall.

The effective branching ratio for a $b \bar{b}$ pair into at least two muons is $6.3 \%$, while the probability for such a pair to be accepted in the present kinematic range is approximately $6 \%$. Correcting appropriately for these factors gives a total cross section for the process $e p \rightarrow b \bar{b} X$ of $11.4 \pm$ $0.8_{-2.9}^{+3.5} \mathrm{nb}$, where the principal systematic uncertainties are from muon reconstruction efficiency, background normalisation and modelling uncertainties. The combined NLO theory calculation gives $7.5_{-2.1}^{+4.5} \mathrm{nb}$, in satisfactory agreement in view of the large uncertainties but inviting a higherorder approach.

\section{References}

[1] ZEUS Collaboration, JHEP 02 (2009) 032.

[2] S. Frixione et al., Nucl. Phys. B 412 (1994) 225;

S. Frixione, P. Nason and G. Ridolfi, Nucl. Phys. B 454 (1995) 3;

M. Cacciari, S. Frixione and P. Nason, JHEP 0103 (2001) 006.

[3] B. Harris and J. Smith, Nucl. Phys. B 454 (1995) 109;

B. Harris and J. Smith, Phys. Lett. B 353 (1995) 535, erratum ibid. B359 (1995) 423;

B. Harris and J. Smith, Phys. Rev. D 57 (1998) 2806. 


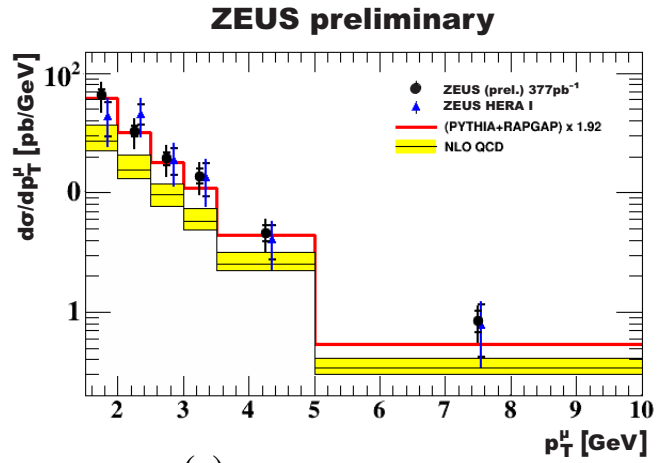

(a)

ZEUS preliminary

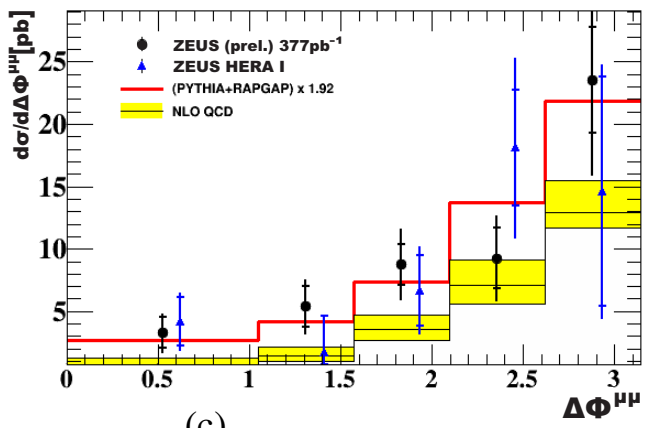

(c)

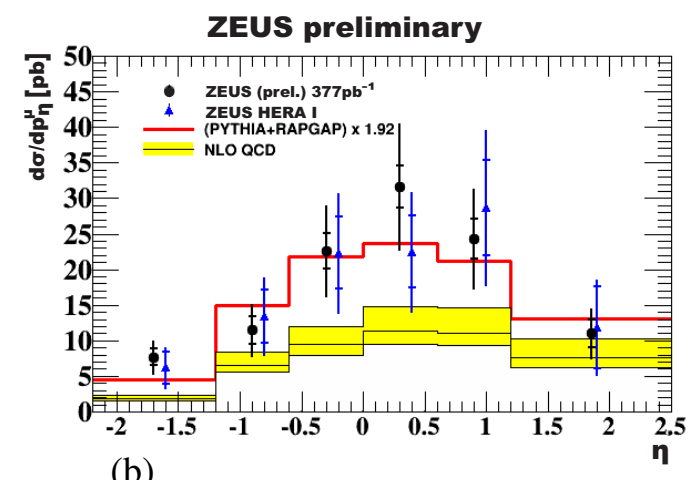

(b)

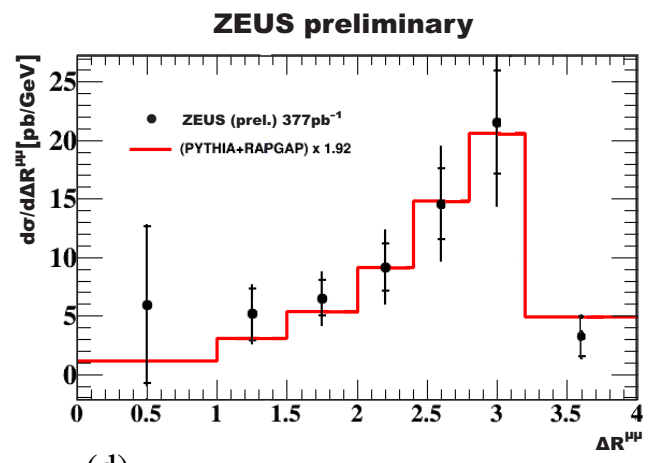

(d)

Figure 2: Differential cross sections for muons from $b$ and $\bar{b}$ decays as functions of (a) muon $p_{T}$ and (b) muon $\eta$. Two muons contribute for each event. Differential cross sections for pairs of muons originating from different $b$ quarks in a $b \bar{b}$ pair, for (c) the azimuthal separation of the muon pair, and (d) the separation $R$ in azimuth and pseudorapidity of the muon pair. Comparisons are given to the NLO theoretical calculation where available and to PYTHIA/RAPGAP. 